(c) 2022 World Scientific Publishing Company

https://doi.org/10.1142/9789811239618_0001

\author{
Chapter 1
}

\title{
The Impact of Multiple Vaccines on the Death Rate - A Focused Review for BCG Vaccination in the COVID-19 Pandemic Period
}

\author{
Sahbi Farhani ${ }^{*, \dagger, * *}$, Imen Gam ${ }^{\ddagger, \S, \dagger \dagger}$, and \\ Ilhem Barboura ${ }^{\pi, 1, \ddagger \ddagger}$ \\ *QUARG UR17ES26, ESCT, Campus University of Manouba, \\ 2010, Manouba, Tunisia \\ ${ }^{\dagger}$ Higher Institute of Finance and Taxation of Sousse, \\ University of Sousse, Sousse, Tunisia \\ ${ }^{\ddagger}$ LAMIDED Laboratory, ISG Sousse, University of Sousse, \\ Sousse, Tunisia \\ ${ }^{\S}$ Higher Institute of Business Administration of Sfax, \\ University of Sfax, Sfax, Tunisia \\ ฯ Biochemistry Laboratory, CHU Farhat Hached, Sousse, \\ Tunisia \\ I Higher School of Health Sciences and Techniques, \\ University of Sousse, Sousse, Tunisia \\ **sahbi.farhani@isffs.u-sousse.tn \\ ††imen.gam@isaas.usf.tn \\ ††ilhembarboura@esstsso.u-sousse.tn
}

Like other infectious diseases, controlling COVID-19 infection must be a priority in order to save all of humanity. In absence of a specific vaccination against the novel 2019 coronavirus, many researchers suggest that Bacillus Calmette-Guérin (BCG) vaccination can boost the human immunity system and have a protective effect against this infection. In this study, we use two methods: Analysis of Covariance (ANCOVA) and 


\begin{abstract}
Ordinary Least Squares (OLS) regression in order to explore the role of existing vaccines on COVID-19 mortality. Results are very promising and interesting. In fact, contrary to the emerging intuition, we prove that BCG vaccination does not have a relevant impact on COVID-19 mortality numbers. In this regards, another contribution of our chapter is showing that other vaccines could be paid more attention, as Hepatitis B of Adults (HepB1), Varicella-Zoster (VZ), and especially, Meningococcal $\mathrm{C}$ conjugate (MenC) vaccines have a negative impact on COVID-19 mortality in contrast to Diphtheria and Tetanus Toxoids (DT) Vac-Cube for older children/adults.
\end{abstract}

Keywords: COVID-19, Vaccines, ANCOVA, OLS Regression

\title{
1. Introduction
}

On December 8th, 2019, the first case of pneumonia disease caused by an unknown virus emerged in Wuhan City, Hubei Province, China. Virological investigation proved that this virus is a singlestranded RNA and it shares a lot of similarity with two others: the Middle East Respiratory Syndrome (henceforth, MERS) viral respiratory infection, also known as camel flu, caused by the MERS-coronavirus (henceforth, MERS-CoV), and the Severe Acute Respiratory Syndrome (henceforth, SARS) as a viral respiratory disease of zoonotic origin caused by SARS-coronavirus (henceforth, SARS-CoV or SARS-CoV-1). That's why, on February 11th, 2020, the World Health Organization (henceforth, WHO) officially named this virus as Coronavirus 2019, also called COVID-19. This disease spread rapidly around the world; thus, we counted until June 2020 more than 210 infected countries, more than 25,664,000 infected persons, and over 855,374 deaths. Obviously, our world is undergoing one of the most severe global health emergencies due to the COVID-19 virus.

Since COVID-19 vaccine is not currently available, Governments have imposed new precautionary measures in order to limit and slow the spread of this pandemic such as social distancing and quarantining. These measures are still insufficient and additional restrictions should be developed. In this context, with adopting another more scientific axis to overcome this disease, many researchers have started to decipher relations between existing vaccines and COVID-19 infection rate. 
Indeed, a major debate has recently emerged concerning the impact of Bacillus Calmette-Guérin (BCG) vaccination on the transmission of COVID-19. Although they are not conclusive and decisive, some works (e.g., Gupta, 2020; Gursel and Gursel, 2020; RedelmanSidi, 2020; Schaaf et al., 2020) tried to identify whether BCG vaccination might have impacted the spread of COVID-19. For his part, Bouhamed (2020) succeeded to develop a prediction model that controlled the evolution of infected and recovered cases using a Deep Learning sequence prediction model and involving four possibilities according to the existence of BCG vaccination in the country and the Tuberculosis (TB) incidence per 100,000 individuals. His result concluded that the suspicions on the BCG vaccination and TB infections rates' implications turned out to be quite relevant.

It is in this framework that our work fits. In fact, the ultimate goal of this study is to clarify the links between existing vaccination and COVID-19. In other words, we seek to identify whether existing vaccination does confer natural protection and participate to limit COVID-19 spread. To achieve our goal, we apply sophisticated data and analysis including two econometric techniques, namely Analysis of Covariance (ANCOVA) and Ordinary Least Squares (OLS).

The remainder of this chapter is structured as follows: Section 2 describes the case of BCG vaccination, Section 3 provides a detailed description of the data used in this study, Section 4 presents the methodological framework and reports the empirical results, and finally Section 5 concludes the chapter.

\section{BCG Vaccination}

Miller et al. (1984) found that countries without universal policies of BCG vaccination (like the cases of Italy and USA) have been more severely affected compared to countries with universal BCG policies. They proposed that BCG vaccination could be attributed the reduced morbidity and mortality in countries with universal BCG policies.

In the same line, several studies have shown protective action of BCG against unrelated respiratory infections both in children and adults. A comparable protection effect of BCG on respiratory infections was shown among the elderly population in Indonesia 
(Rosenberg et al., 2020). Prospective clinical trial performed in Japan has shown BCG vaccine to protect from pneumonia in tuberculin-negative elderly populations (Gao et al., 2020). Randomized controlled trials have demonstrated that the BCG vaccine has immunomodulatory effects to protect partially against respiratory infections. In South Africa, BCG-Danish reduced respiratory tract infections by $73 \%$ (95\% CI, 39 to 88) in adolescents (Curtis et al., 2020). Ozdemir et al. (2020) have shown proportionately less cases, milder illness, and a lower death rate in BCG vaccinated population as compared to BCG non-vaccinated across countries and hemispheres. According to Madan et al. (2020), BCG vaccination might alter a secondary innate immune response upon viral infection over a month apart resulting in improved antiviral responses and lowering viremia. Thus, it is so far proven that the countries more prone to be severely affected by SARS-CoV-2 did not adopt universal policies of BCG vaccination, like Italy and Spain. A reasonable explanation is that children of the country where routine childhood $B C G$ vaccination is a policy and $B C G$ vaccinated children have some degree of protection from infection from SARS-CoV-2 as well as less severe diseases among those who had been infected.

On the contrary, in particular work, Hamiel et al. (2020) studied the current policy of the Israeli Ministry of Health that tested for SARS-CoV-2 in every patient with symptoms that could be compatible with COVID-19 (cough, dyspnea, fever, etc.). Empirically, Chi-Square $\left(\chi^{2}\right)$ tests were used to compare proportions and rates per 100,000 population of positive test results among persons with symptoms compatible with COVID-19 born from 1979 to 1981 (aged 39-41 years) with those born from 1983 to 1985 (aged 35-37 years). A two-sided significance threshold was set at $P$-value less than 0.05 (typically $P \leq 0.05$ ). Of 72,060 test results reviewed, 3,064 were from patients born between 1979 and 1981 and 2,869 were among likely unvaccinated people born between 1983 and 1985. There was no statistically significant difference in the proportion of positive test results in the BCG-vaccinated group (361 [11.7\%]) versus the unvaccinated group (299 [10.4\%]; difference, $1.3 \%$; $95 \%$ confidence interval [CI],$-0.3 \%$ to $2.9 \% ; P=0.09)$ or in positivity rates per 100,000 (121 in vaccinated group versus 100 in unvaccinated group; difference, 21 per 100,000; $95 \%$ CI, -10 to 50 per 100,$000 ; P=0.15)$. There was one case of severe disease (mechanical ventilation or intensive care 
unit admission) in each group, and no deaths were reported. This study does not support the idea that BCG vaccination in childhood has a protective effect against COVID-19 in adulthood.

In the same line, the WHO (2020) affirmed that there is no evidence that BCG protects people against infection with the COVID-19 virus. Several studies have compared the incidence of COVID-19 in countries where the BCG vaccine is used and have observed that reported cases of COVID-19 were lower in countries where the new infants are systematically vaccinated against TB. According to the Belgian medical news site,${ }^{1,2}$ the $B C G$ vaccine does not protect directly from COVID-19 but could provide a boost to the immune system. However, other factors must be taken into account such as the difference between national demographics, the morbidity rate, the COVID-19 screening rate, and the stage of the pandemic in each country, in order to specify that these studies were published in preprint, that is to say without peer validation (Ministry of Health, Labour, and Welfare of Japan, 2020).

\section{Data}

Our chapter adds to the existing literature in different ways. The first contribution stems from the fact that we compile a huge database encompassing an important number of indicators covering 175 countries (see Appendix). All data are obtained from the World Bank and the WHO Websites. Indeed, we consider a set of 35 variables as follows:

\section{1st Type: Dependent variable}

Number of deaths per 100 infections $(\boldsymbol{N D})$ : this variable represents the evolution of deaths due to COVID-19 infections for 175 countries from the disease onset in each country until June 6, 2020.

\footnotetext{
${ }^{1}$ WHO (2020). Immunization in the context of COVID-19 pandemic. Frequently Asked Questions (FAQ). April 16, 2020. Available at: https://apps. who.int/iris/bitstream/handle/10665/331818/WHO-2019-nCoV-immunization_s ervices-FAQ-2020.

${ }^{2}$ WHO (2020). BCG vaccination and COVID-19. Scientific Brief. April 12, 2020. Available at: https://www.who.int/news-room/commentaries/detail/bacille-c almette-gu\% C3\% A9rin-(bcg)-vaccination-and-covid- 19 .
} 
2nd Type: Quantitative variables

- Percent of Population more than 65 years (Pop) as a demographic indicator: it turns out that individuals who are elderly register the highest death risk from COVID-19.

\begin{tabular}{|c|c|c|}
\hline & $\begin{array}{l}\text { Qualitative Independent } \\
\text { Variables (Vaccines) }\end{array}$ & Abbreviation \\
\hline 1 & Bacillus Calmette-Guérin & BCG \\
\hline 2 & Diphtheria and Tetanus & $\mathrm{DT}$ \\
\hline 3 & Diphtheria, Tetanus, Pertussis & $\mathrm{DTaP}$ \\
\hline 4 & Diphtheria and Tetanus toxoids and whole-cell Pertussis & DTwP \\
\hline 5 & $\begin{array}{l}\text { Diphtheria, Tetanus, Pertussis, Hepatitis B, Polio, and } \\
\text { Haemophilus influenza type b (DTaP-HB-IPV-Hib) }\end{array}$ & DTHIHB \\
\hline 6 & $\begin{array}{l}\text { Diphtheria and Tetanus Toxoid with Pertussis, Hib and } \\
\text { HepB (DTwPHibHepB) }\end{array}$ & DTТРНHР \\
\hline 7 & Hepatitis A for Adult (HepA_Adult) & HepA1 \\
\hline 8 & Hepatitis A for Child (HepA_Pediatric) & HepA2 \\
\hline 9 & Hepatitis B for Adult (HepB_Adult) & HepB1 \\
\hline 10 & Hepatitis B for Child (HepB_Pediatric) & HepB2 \\
\hline 11 & Haemophilus influenza type b & Hib \\
\hline 12 & Human papillomavirus & $\mathrm{HPV}$ \\
\hline 13 & Influenza Vaccine (Flu Vaccine)-Adult & IV1 \\
\hline 14 & Influenza Vaccine (Flu Vaccine)-Pediatric & IV2 \\
\hline 15 & Inactivated Polio Vaccine & IPV \\
\hline 16 & Measles & Measles \\
\hline 17 & $\begin{array}{l}\text { Meningococcal ACY and W-135 diphtheria conjugate } \\
\text { (MenACWY-135 conj) }\end{array}$ & MenACWY135 \\
\hline 18 & Meningococcal A conjugate (MenA_conj) & MenA \\
\hline 19 & Meningococcal C conjugate (MenC_conj) & MenC \\
\hline 20 & Measles, Mumps, and Rubella & MMR \\
\hline 21 & Measles, Mumps, Rubella, and Varicella & MMRV \\
\hline 22 & Measles-Rubella & $\mathrm{MR}$ \\
\hline 23 & Oral Polio Vaccine & $\mathrm{OPV}$ \\
\hline 24 & Pneumococcal Conjugate Vaccine & $\mathrm{PCV}$ \\
\hline 25 & Pneumoccocal Polysacharide Vaccine (Pneumo_Ps) & PPV \\
\hline 26 & Rotavirus & Rotavirus \\
\hline 27 & Tetanus-Diphtheria & $\mathrm{TD}$ \\
\hline 28 & Tetanus, Diphtheria, Pertussis & Tdap \\
\hline 29 & Tetanus Toxoid & $\mathrm{TT}$ \\
\hline 30 & Vitamin A & VitaminA \\
\hline 31 & Yellow Fever & YF \\
\hline 32 & Varicella-Zoster & $\mathrm{VZ}$ \\
\hline
\end{tabular}


- Crude death rate per 1,000 in $2018(\boldsymbol{C D R})$ as a medical indicator: this variable is used to approximate the general health status by countries. In fact, due to a lack of data, we cannot collect a database concerning the mortality number per type of disease by country. In fact, many researchers prove that individuals with chronic health problems have a higher risk of mortality due to the COVID-19 infection.

3rd Type: Qualitative variables representing medical indicators, especially vaccination

These variables are binary. They take 1 if the vaccine is available in the country under consideration and 0 if not.

\section{Methods and Results}

Our empirical analysis is used to answer the following question: Could an existing vaccination adopted by the different countries influence the number of deaths due to COVID-19?

In other words, we are looking for the existence of significant links between vaccination and COVID-19 deaths. To reach our objective, we adopt two techniques: ANCOVA and OLS. A vaccine is considered as a powerful vector to control COVID-19 mortality if it is judged significant by the two techniques mentioned above.

To our best knowledge, there is hardly any research been done about the evolution of number of deaths per 100 COVID-19 infections involving this huge number of variables and countries and using these techniques.

In the rest of this part of our work, we apply a bottom-up scheme starting with the general data analysis to subsequently arrive at a more sophisticated and precise econometric analysis.

\subsection{Covariance analysis (ANCOVA)}

ANCOVA is a general linear model which combines Analysis of variance (ANOVA) and regression. It is considered among the most suitable techniques in presence of a quantitative endogenous variable 
and mixed (qualitative and quantitative) exogenous ones. More precisely, it is used to examine if the mean values of the dependent variables are equal across levels of categorical exogenous variables, while taking into account the influence of other continuous independent factors, known as covariates. Mathematically, ANCOVA decomposes the variance of the dependent variable into three main parts: variance explained by qualitative independent variables, variance explained by covariates, and residual variance. The general model of the analysis of covariance is formulated as follows:

$$
Y_{i j}=\mu+\alpha_{i}+\beta\left(X_{i j}-\bar{X}_{i}\right)+\varepsilon_{i j}
$$

where

- $i$ is the category index of the categorical variable and $j$ is the index of observations in each category;

- $Y_{i j}$ is the $j t h$ observation under the ith categorical group for the dependent variable;

- $X_{i j}$ represents the $j t h$ observation of the covariate under the ith group;

- $\mu$ is the general mean;

- $\alpha_{i}$ denotes the difference between the average of group $i$ and the general average;

- $\beta$ is the slope of the regression of the dependent variable on the quantitative independent variable;

- $\bar{X}_{i}$ is the mean of the quantitative independent variable for category $i$;

- $\varepsilon_{i j}$ denotes the error term.

The premises of ANCOVA are also borrowed from those of ANOVA and linear regression:

- The relationship between the endogenous variable and the exogenous variables must be linear.

- The slope of this relation must be equal for the different modalities of the variable.

- The residues are independent and normally distributed.

- The residuals variance is homogeneous for the different modalities of the independent variable.

The estimation of Eq. (1) using ANCOVA method is summarized in Table 1. 
Table 1: ANCOVA estimation results (Number of death, ND as a dependent variable).

\begin{tabular}{llll}
\hline Variables & Significance Test & Variables & Significance Test \\
\hline Corrected Model & $\mathbf{. 0 1 6 * *}$ & IPV & .452 \\
Intercept &. $\mathbf{0 4 6}^{* *}$ & Measles & .228 \\
Pop & .357 & MenACWY135 & .574 \\
CDR & .452 & MenA & .178 \\
BCG & .502 & MenC & $\mathbf{. 0 5 7 * *}$ \\
DT & .284 & MMR & .920 \\
DtaP & .980 & MMRV & .285 \\
DTwP & .750 & MR & .826 \\
DTHIHB & .699 & OPV & .444 \\
DTTPHHB & .682 & PCV & .719 \\
HepA1 & .637 & PPV & .278 \\
HepA2 & .962 & Rotavirus & .403 \\
HepB1 & $.033^{* *}$ & TD & $.096 * *$ \\
HepB2 & .594 & Tdap & .569 \\
Hib & .180 & TT & .590 \\
HPV & .308 & VitaminA & .824 \\
IV1 & .653 & YF & .929 \\
IV2 & .633 & Varicella & $.095 * *$ \\
\hline
\end{tabular}

Note: ${ }^{* *}$ and $* * *$ indicate the significance at the thresholds of 5 and $10 \%$, respectively.

Contrary to the doubts advanced in the existing literature, ANCOVA approach proves that BCG vaccination has no effect on COVID-19 mortality. Contrariwise, Hepatitis B-Adult (HepB1), MenC-conjugate (MenC), Tetanus-Diphtheria for older children/adults Vac-Cube (TD), and Varicella vaccination seems to be significant since their $P$-values are under the statistical level of 5 and $10 \%$. Moreover, the model is globally significant since the $P$-value of the corrected model does not exceed the threshold of $5 \%$. To be effective, the vaccine chosen must be immunogenic and well preserved between the subspecies of the target pathogen, in order to confer sufficient immunity.

In order to complete this analysis, we proceed to estimate the Number of deaths per 100 (ND) COVID-19 infections by adopting the OLS approach. This analysis is essential in order to ensure the significance of the variables highlighted by the ANCOVA approach as 
well as to determine the direction and height of the response following the stimulation of an explanatory variable.

\subsection{OLS regression}

In statistics, OLS regression is the most common estimation method and widely used to estimate the relationship between one or more exogenous qualitative or quantitative variables and the endogenous quantitative one as explained by the following formula:

$$
y_{i}=\alpha+\beta_{1} x_{1}+\beta_{2} x_{2}+\cdots+\beta_{n} x_{n}+\varepsilon_{i}
$$

where $\varepsilon_{i}$ is an error term, $\alpha$ represents the constant term, and $\beta_{i}$ represents the direct elasticities with respect to the different independent variables.

This method relies on the minimization of the sum of the squares in the difference between observed and estimated values of the endogenous variables.

Although it is used especially in the field of consumption analysis, our work is the only one that proposes OLS regression to inspect COVID-19 mortality. In fact, we will analyze in our chapter the following model:

$$
\begin{aligned}
\mathrm{ND}_{i}= & \alpha+\beta_{1} \text { Pop }_{\mathrm{i}}+\beta_{2} \mathrm{CDR}_{\mathrm{i}}+\beta_{3} \mathrm{BCG}_{\mathrm{i}}+\beta_{4} \mathrm{DT}_{\mathrm{i}}+\beta_{5} \mathrm{DTaP}_{\mathrm{i}} \\
& +\beta_{6} \mathrm{DTwP}_{\mathrm{i}}+\beta_{7} \mathrm{DTHIHB}_{\mathrm{i}}+\beta_{8} \mathrm{DTTPHHB}_{\mathrm{i}}+\beta_{9} \mathrm{HepA}_{\mathrm{i}} \\
& +\beta_{10} \mathrm{HepA}_{\mathrm{i}}+\beta_{11} \mathrm{HepB}_{\mathrm{i}}+\beta_{12} \mathrm{HepB}_{\mathrm{i}}+\beta_{13} \mathrm{HIB}_{\mathrm{i}}+\beta_{14} \mathrm{HPV}_{\mathrm{i}} \\
& +\beta_{15} \mathrm{IV}_{\mathrm{i}}+\beta_{16} \mathrm{IV}_{\mathrm{i}}+\beta_{17} \mathrm{IPV}_{\mathrm{i}}+\beta_{18} \text { Measles }_{\mathrm{i}} \\
& +\beta_{19} \mathrm{MenACWY}_{3} 5_{\mathrm{i}}+\beta_{20} \mathrm{MenA}_{\mathrm{i}}+\beta_{21} \mathrm{MenC}_{\mathrm{i}} \\
& +\beta_{22} \mathrm{MMR}_{\mathrm{i}}+\beta_{23} \mathrm{MMRV}_{\mathrm{i}}+\beta_{24} \mathrm{MR}_{\mathrm{i}}+\beta_{25} \mathrm{OPV}_{\mathrm{i}} \\
& +\beta_{26} \mathrm{PCV}_{\mathrm{i}}+\beta_{27} \mathrm{PPV}_{\mathrm{i}}+\beta_{28} \text { Rotavirus }_{\mathrm{i}}+\beta_{29} \mathrm{TD}_{\mathrm{i}} \\
& +\beta_{30} \text { Tdap }_{\mathrm{i}}+\beta_{31} \mathrm{TT}_{\mathrm{i}}+\beta_{32} \text { VitaminA }_{\mathrm{i}}+\beta_{33} \mathrm{YF}_{\mathrm{i}} \\
& +\beta_{34} \text { Varicella }_{\mathrm{i}}+\varepsilon_{\mathrm{i}}
\end{aligned}
$$

where $i$ indicates the different countries under consideration in our empirical investigation.

Results of OLS regression are represented in Table 2. Evidently, OLS regression estimation has just confirmed results obtained using ANCOVA method. Indeed, our analysis uncovers three stylized facts. 
Table 2: OLS estimation results (Number of deaths, ND, as a dependent variable).

\begin{tabular}{lcclrl}
\hline Variables & Coef. & $\boldsymbol{p}$-value & Variables & Coef. & $\boldsymbol{p}$-value \\
\hline Intercept & 1.933 & .812 & IPV & -.696 & .452 \\
& & & Measles & 1.259 & .228 \\
Pop & .096 & .357 & MenACWY135 & .779 & .574 \\
CDR & .121 & .452 & MenA & -2.302 & .178 \\
BCG & .693 & .502 & MenC & $-\mathbf{2 . 5 0 0}$ & $\mathbf{. 0 5 7 * *}$ \\
DT & .859 & .284 & MMR & -.120 & .920 \\
DtaP & .022 & .980 & MMRV & 1.733 & .285 \\
DTwP & -.271 & .750 & MR & .207 & .826 \\
DTHIHB & .420 & .699 & OPV & .931 & .444 \\
DTTPHHB & .568 & .682 & PCV & .284 & .719 \\
HepA1 & -.830 & .637 & PPV & 1.102 & .278 \\
HepA2 & .056 & .962 & Rotavirus & -.595 & .403 \\
HepB1 & $-\mathbf{2 . 2 7 6}$ & $\mathbf{. 0 3 3} *$ TD & $\mathbf{1 . 3 1 3}$ & $\mathbf{. 0 9 6 * * *}$ \\
HepB2 & .385 & .594 & Tdap & -.525 & .569 \\
Hib & 1.233 & .180 & TT & .432 & .590 \\
HPV & .720 & .308 & VitaminA & -.169 & .824 \\
IV1 & -.704 & .653 & YF & -.078 & .929 \\
IV2 & .694 & .633 & Varicella & $-\mathbf{1 . 5 6 8}$ & $\mathbf{. 0 9 5 * * *}$ \\
\hline
\end{tabular}

Note: ${ }^{* *}$ and $* * *$ indicate the significance at the thresholds of 5 , and $10 \%$, respectively.

First, our investigation refutes the doubts of the protection provided by BCG vaccination against the mortality caused by COVID-19 infection. Second, OLS regression proves that three vaccines have a significant negative impact on the mortality number caused by COVID-19 disease. In fact, the $P$-value of HepB1 variable is under the statistical thresholds of $5 \%$ confirming, thus, that the number of deaths per 100 COVID-19 infections decreases by $2.276 \%$ for countries adopting Hepatitis B-Adult vaccine. This means that this vaccine is an inert peptide vaccine, which consists of only one or more proteins of the pathogen. However, in order to be effective, the peptides chosen must be immunogenic and well preserved between the subspecies of the target pathogen, in order to confer sufficient immunity (Alleman et al., 2018).

Similarly, MenC and Varicella variables are statistically significant at the $10 \%$ level proving that COVID-19 mortality decline, 
respectively, by $2.5 \%$ and $1.568 \%$ for countries providing MenCconjugate and Varicella vaccines to their citizens. In addition, we can also suggest that Varicella vaccination is an attenuated live vaccine essentially produced from attenuated viral strains by successive passages in culture on cells competent for viral replication. Since these vaccine strains are replicative, they perfectly mimic infection by the pathogenic germ in the vaccinated subject, which gives them excellent immunogenicity, in particular by activating innate immunity and CD8+ T lymphocytes and by inducing a prolonged memory response (Paul and Lelièvre, 2018). From this analysis, we can conclude that Hepatitis B adult, MenC-conjugate, and Varicella vaccination can boost immune system against COVID-19 infections. Another explanation to this result lies in the fact that the use of these vaccines can intervene to avoid the infection by chronic and severe diseases and subsequently complicate the situation more in case of COVID-19 infection.

Finally, contrary to our suspicions, it seems that TetanusDiphtheria (TD) vaccine has a positive and significant impact on mortality number due to COVID-19 disease at the 10\% statistical level, with an increase of $1.313 \%$.

In general, these vaccines are not very immunogenic because, by being reduced to only protective antigenic epitopes, they do not contain an agent able to amplify and orient innate immune responses then be adaptive. They require therefore the addition of adjuvants to be effective. They require also regular booster injections because they induce a short-lived memory response. However, the counterpart of this lack of efficiency is increased job security, with unrestricted use in immunocompromised subjects and very few side effects (Goullé and Grangeot-Keros, 2020).

\subsection{Other factors}

Some other factors are considered is this study, such as: (i) the number of days from diagnosis of the first patient with COVID-19 infection in each prefecture up to March 29, 2020; (ii) the populations that migrated to and from each prefecture in 2018 and which obtained from the annual report on internal migration in Japan derived from the basic resident registration; (iii) Inhabitable area that is calculated using data of the Statistics Bureau, Ministry of Internal Affairs and Communications; and (iv) the ratio of day to nighttime population, 
the ratio of households of five individuals or more to entire households, and the ratio of workers in the primary, secondary, and tertiary sectors of industry to the entire working population that was obtained from results of the national Census in 2015.

\section{Conclusion}

In this study, by applying ANCOVA and OLS regression on 35 variables and 175 countries, we tried to highlight the main vectors, especially among existing vaccinations, that influence the number of deaths due to COVID-19 infections. Evidently, we register a consensus between the two techniques about the role that Hepatitis B for Adult, MenC-conjugate, Varicella vaccine play in the reduction of COVID-19 mortality.

Contrary to our doubts, we reject the underlining hypothesis that BCG vaccine can be protective against COVID-19 mortality since only two techniques confirm it. This result does not invalidate that $\mathrm{BCG}$ vaccine might have impacted the transmission of COVID-19 infections. In addition, these vaccines are more purveyors of side effects than the others, and can in particular induce vaccine pathologies similar to the pathology against which they are supposed to protect in immunocompromised subjects (secondarily or constitutive). They are therefore formally contraindicated in these patients, as well as, as a precaution, in pregnant women (Alleman et al., 2018).

So the side effects drastically and scientifically documented remain exceptional. The benefit-risk ratio is in favor of vaccines, and these have shown their effectiveness in reducing or even eradicating the transmission of pathogens associated with major morbidity and mortality. The emergence of new pathogens, and understanding the mechanisms of resistance to certain infectious agents, such as COVID-19, make it necessary to develop new vaccines and new adjuvants, but also new types of vaccines (DNA vaccines, viral pseudo-particles, viral vectors,...) which could allow new modes of activation of the immune system.

The findings arising from this analysis could help public health professionals and decision-makers to take the right decisions and procedures in order to stand up to this pandemic. 


\section{References}

Alleman, M. M., Chitale, R., Burns, C. C. et al. (2018). Vaccine-derived poliovirus outbreaks and events - three provinces, Democratic Republic of the Congo, 2017. Morbidity and Mortality Weekly Report, 67, pp. 300-305.

Bouhamed, H. (2020). COVID-19, Bacille Calmette-Guérin (BCG) and Tuberculosis: Cases and recovery previsions with deep learning sequence prediction. Ingénierie des Systèmes d'Information, 25(2), pp. $165-172$.

Curtis, N., Sparrow, A., Ghebreyesus, T. A. and Netea, M. G. (2020). Considering BCG vaccination to reduce the impact of COVID-19. Lancet, 395(10236), pp. 1545-1546.

Gao, J., Tian, Z. and Yang, X. (2020). Breakthrough: Chloroquine phosphate has shown apparent efficacy in treatment of COVID-19 associated pneumonia in clinical studies. BioScience Trends, 14(1), pp. $72-73$.

Goullé, J. P. and Grangeot-Keros, L. (2020). Aluminum and vaccines: Current state of knowledge. Médecine et Maladies Infectieuses, 50(1), pp. 16-21.

Gupta, A. (2020). Is immuno-modulation the key to COVID-19 pandemic? Indian Journal of Orthopaedics, 54, pp. 394-397.

Gursel, M. and Gursel, I. (2020). Is global BCG vaccination-induced trained immunity relevant to the progression of SARS-CoV-2 pandemic? Allergy, 75(7), pp. 1815-1819.

Hamiel, U., Kozer, E. and Youngster, I. (2020). SARS-CoV-2 rates in BCG-vaccinated and unvaccinated young adults. JAMA, 323(22), pp. 2340-2341.

Madan, M., Pahuja, S., Mohan, A. et al. (2020). TB infection and BCG vaccination: are we protected from COVID-19? Public Health, 185, pp. 91-92.

Miller, C. L., Morris, J. and Pollock, T. M. (1984). PHLS inquiry into current BCG vaccination policy. British Medical Journal (Clinical Research Edition), 288(6416), p. 564.

Ministry of Health, Labour and Welfare of Japan. (2020). About Coronavirus Disease 2019 (COVID-19). https://www.mhlw.go.jp/stf/seisa kunitsuite/bunya/0000164708_00001.html.

Ozdemir, C., Kucuksezer, U. C. and Tamay, Z. U. (2020). Is BCG vaccination affecting the spread and severity of COVID-19? Allergy, 75(7), pp. 1824-1827.

Paul, S. and Lelièvre, J. D. (2018). Immunologie fondamentale et immunopathologie. In: Le mécanisme d'action des vaccins, le rôle 
des adjuvants, Eds. Collège des enseignants d'immunologie, 2nd ed., Paris: Elsevier Masson, pp. 255-262.

Redelman-Sidi, G. (2020). Could BCG be used to protect against COVID19? Nature Reviews Urology, 17(6), pp. 316-317.

Rosenberg, E. S., Dufort, E. M., Udo, T. et al. (2020). Association of treatment with hydroxychloroquine or azithromycin with in-hospital mortality in patients with COVID-19 in New York State. JAMA, 323(24), pp. 2493-2502.

Schaaf, H. S., du Preez, K., Kruger, M. et al. (2020). Bacille CalmetteGuérin (BCG) vaccine and the COVID-19 pandemic: Responsible stewardship is needed. The International Journal of Tuberculosis and Lung Disease, 24(7), pp. 732-734.

WHO. (2020). Joining forces to end TB and COVID-19. https://www.who .int/news/item/12-05-2020-joining-forces-to-end-tb-and-covid-19. 


\section{Appendix}

\begin{tabular}{|c|c|c|c|c|c|c|}
\hline Afghanistan & Bulgaria & Equatorial Guinea & Iraq & Mauritania & Philippines & Sri Lanka \\
\hline Albania & Burkina Faso & Eritrea & Iran & Mauritius & Poland & Sudan \\
\hline Algeria & Burundi & Estonia & Ireland & Mexico & Portugal & Suriname \\
\hline Andorra & Cabo Verde & Eswatini & Italy & Moldova Rep. & Qatar & Sweden \\
\hline Angola & Cambodia & Ethiopia & Ivory Coast & Monaco & Romania & Switzerland \\
\hline $\begin{array}{l}\text { Antigua and } \\
\text { Barbuda }\end{array}$ & Cameroon & Fiji & Jamaica & Mongolia & Russia & Syria \\
\hline Argentina & Canada & Finland & Japan & Montenegro & Rwanda & Tajikistan \\
\hline Armenia & Chad & France & Jordan & Morocco & Saint Lucia & Tanzania \\
\hline Australia & Chile & Gabon & Kazakhstan & Mozambique & $\begin{array}{l}\text { Saint Vincent } \\
\text { and Grenadines }\end{array}$ & Thailand \\
\hline Austria & China & Gambia & Kenya & Myanmar & Salvador & Timor-Leste \\
\hline Azebaijan & Colombia & Georgia & Kuwait & Namibia & San Marino & Togo \\
\hline Bahamas & Comoros & Germany & Kyrgyzstan & Nepal & $\begin{array}{l}\text { Sao Tome and } \\
\text { Principe }\end{array}$ & $\begin{array}{l}\text { Trinidad and Tobago } \\
\text { Tobago }\end{array}$ \\
\hline Bahrain & Congo & Ghana & Latvia & Netherlands & Saudi Arabia & Tunisia \\
\hline Bangladesh & Costa Rica & Greece & Lebanon & New Zealand & Senegal & Turkey \\
\hline Barbados & Croatia & Grenada & Lesotho & Nicaragua & Serbia & Uganda \\
\hline Belarus & Cuba & Guatemala & Liberia & Niger & Seychelles & $\mathrm{UK}$ \\
\hline Belgium & Cyprus & Guinea & Libya & Nigeria & Sierra Leone & Ukraine \\
\hline Belize & Czech Rep. & Guinea-Bissau & Lithuania & $\begin{array}{l}\text { North } \\
\text { Macedonia }\end{array}$ & Singapore & Uruguay \\
\hline Benin & Denmark & Guyana & Luxembourg & Norway & Slovakia & USA \\
\hline Bhutan & Djibouti & Haiti & Madagascar & Oman & Slovenia & Uzbekistan \\
\hline Bolivia & Dominica & Honduras & Malawi & Pakistan & Somalia & Venezuela \\
\hline $\begin{array}{l}\text { Bosnia and } \\
\text { Herzegovina }\end{array}$ & Dominican Rep. & Hungary & Malaysia & Panama & South Africa & Vietnam \\
\hline Botswana & Ecuador & Iceland & Maldives & $\begin{array}{l}\text { Papua New } \\
\text { Guinea }\end{array}$ & South Korea & Yemen \\
\hline Brazil & Egypt & India & Mali & Paraguay & South Sudan & Zambia \\
\hline $\begin{array}{l}\text { Brunei } \\
\text { Darussalam }\end{array}$ & Emirate & Indonesia & Malta & Peru & Spain & Zimbabwe \\
\hline
\end{tabular}

iantan

Azebaijan

Bhutan

Bosnia and

Herzegovina

Brazil

Daruss
Chad

China

Colombia

Congo

Costa Rica

roatia

Cyprus

Dominica

Haiti

Hungary

India

Indonesia
Ir

Ivory Coast

Japan

Jordan

rgyzstan

Lesotho

Liberia

Libya

Luxembourg

Madagascar

Malaw

Mali

Malta
Maurit

Monaco

Montenegro

Morocco

New Zealand

caragua

Nigeria

Oman

Pakistan

Papua New

Paraguay 